5 Ward, J N, Draper, J W, and Lavengood, R W, fournal of Urology, 1967, 98, 48.

${ }^{6}$ Halpern, M H, Dalrymple, G, and Young, J, fournal of Urology, 1970, 103,21 .

${ }^{7}$ Hatfield, P M, and Pfister, R C, Fournal of the American Medical Association, 1972, 222, 1527.

${ }^{8}$ Kendall, A R, Pollack, H M, and Karafin, L, Urology, 1974, 4, 635.

- Keith, N M, Wagener, H P, and Barker, N W, American fournal of Medical Science, 1939, 197, 332.

10 Williams, G, Mitcheson, H D, and Castro, J E, in press.
11 Wolfson, M, et al, Dialysis and Transplantation, 1976, 6, 66.

12 Lazarus, J E, et al, Fournal of the American Medical Association, 1971, 217, 1821.

13 Salvatierra, O, Kountz, S L, and Belzer, P O, Surgery, Gynaecology and Obstetrics, 1973, 137, 431.

${ }^{14}$ Mendez, R, et al, Urology, 1975, 5, 26.

15 Fournal of the American Medical Association, 1975, 233, 787.

16 Oettinger, C W, et al, New England fournal of Medicine, 1974, 291, 458.

\title{
The nurse's role in immediate postoperative care
}

\author{
D S B STEPHENS, J BOALER
}

British Medical fournal, 1977, 1, 1199-1202

\begin{abstract}
Summary
From the time that a patient leaves the care of the anaesthetist after an operation until he wakes in the ward his physiological state should be continuously and expertly supervised. Postoperative nurses are provided only when the operating theatre has a recovery room. A survey among consultants and nurses in one region showed that many surgical units did not have recovery rooms and that inexperienced ward nurses were often sent to collect patients. The survey showed that most nurses were competent to care for unconscious patients so long as an emergency did not arise. In many hospitals the facilities for the safe nursing of postoperative patients were totally inadequate.

The very least that is needed is good communications with the anaesthetist, adequate lighting, and a source of oxygen and suction. Because of the shortage of nurses it would be realistic to recognise that trainee nurses are likely to have to care for postanaesthetic patients early on and to train them accordingly. Nevertheless, recovery nurses, whose sole responsibility is to care for a patient until he has recovered from anaesthesia, should be appointed for all busy surgical units.
\end{abstract}

\section{Introduction}

In parts of Europe and the United States of America specially trained nurses take an active though limited part in the conduct of anaesthetics. In the United Kingdom they play no part in the specialty. British anaesthetists defend this anomalous position by maintaining that nurses are not qualified to accept the responsibilities that the specialty demands. Yet immediately the patient leaves the operating theatre his care passes into the hands of a nurse, who may be a fully trained sister or a first-year trainee. Although the anaesthetist remains responsible for the patient, he is invariably not readily available to deal with any emergency that may arise in the patient who has just passed out of his hands.

It is a tribute to modern anaesthesia and postoperative nursing care that only about 100 unexplained deaths associated with anaesthesia occurred yearly in $1959-70,{ }^{1}$ while over 4000000

Medway Hospitals Group, Kent

D S B STEPHENS, MB, FFARCS, consultant anaesthetist

J BOALER, DOBSTRCOG, FFARCS, consultant anaesthetist anaesthetics were given each year in England and Wales alone. The Registrar General's figures do not identify the stage when death occurred, nor do they distinguish between avoidable and unavoidable deaths. Wylie recently examined the Medical Defence Union records of the past 20 years and reported that about a third of the complications associated with anaesthesia occurred in the postoperative period, about half of these culminating in cardiac arrest. ${ }^{2}$ These figures suggest that failure in postoperative management may have been responsible.

Immediately after operation patients are exposed to certain risks such as regurgitation and vomiting, obstruction of the respiratory passages, and cardiac insufficiency. Well-trained staff and adequate resuscitation equipment are essential for treating, and whenever possible preventing, these complications. The equipment is best provided in a modern recovery room within easy reach of the anaesthetist. ${ }^{3}$ Theatre nurses are officially provided to care for postanaesthetic patients only if there is a recovery room attached to the theatre. If there is no such room the theatre nurse usually relinquishes her responsibility for the patient to a ward nurse as soon as possible. The ward nurse may be inexperienced, frightened, and uncertain of her role in these circumstances. And, because there is no recovery room, she may have to care for the patient in a poorly lit, draughty corridor while the anaesthetist is by this time busy with the next case. The continuing shortage of nurses, the ever-increasing volume of surgery, ${ }^{4}$ and the lack of special equipment are making it increasingly difficult to provide adequate care in the critical postanaesthetic period.

Most anaesthetists are aware of these problems and modify their techniques so that patients will wake promptly at the end of surgery and rapidly regain their reflexes. This is not always in the best interests of the patient, who might benefit from a peaceful recovery in a safe environment. The patients who take the longest time to wake are those who have had an inhalation anaesthetic (as distinct from a muscle relaxant artificial ventilation technique) lasting half an hour or more. These anaesthetics are given for such common and relatively simple procedures as herniorrhaphy and varicose vein surgery in otherwise fit people, and it is often these patients who unexpectedly provide the most problems in the recovery period.

Although they are trained in their part in the management of cardiorespiratory arrest, nurses are not generally sufficiently aware of the particular complications that arise in the immediate postoperative period and how they can be prevented. Records of complications, even of cardiac arrest, are often inadequate, and in many cases nothing is recorded in the patient's notes if resuscitation has been successful.

We decided to investigate this further, concentrating on nursing knowledge and competence and on the facilities provided for postoperative recovery in a cross-section of hospitals in this region. 


\section{Nursing knowledge and experience}

One hundred and sixty-three nurses in one health district were asked to complete a confidential questionnaire to assess their knowledge and attitudes to caring for patients immediately after operation. The questionnaire was constructed with the advice of senior clinial and tutorial nurses. The nurses were randomly selected but we tried to have roughly equal numbers in each of four categories: trained State-registered nurses (SRNs) and trained State-enrolled nurses (SENs) (group 1), and student nurses and pupil nurses (group 2). Only trainees with over six months' training were included, as we assumed that less experienced trainees would not be expected to take responsibility for patients in the early postoperative period. Each nurse was interviewed individually by one of us (DSBS or JB). We provided individual help and guidance when appropriate, and the element of nervous tension that accompanies any test was thereby minimised. Each nurse was asked to provide details of his or her experience and what training he or she had received in the problems likely to be encountered in the postoperative period. The questions are shown in fig 1 .

(1) What are the two important things a nurse does on taking over an unconscious patient after surgery under a general anaesthetic?

(2) What three observations should be carried out on the patient?

(3) What is the ideal position of an unconscious patient after a general anaesthetic?

(4) What three things would you do if the patient stopped breathing ?

(5) What three things would you do if the patient vomited or regurgitated?

(6) What difficulties would you expect with:

(a) Obese patients?

(b) Coloured patients?

(7) At what time do you think the patient is ready to return to the ward ? (a) When fully conscious?

(b) When the airway has been ejected?

(c) While still unconscious-that is, immediately?

(8) If you were the only nurse on the ward and one patient was unconscious describe how you would care for this patient and also other patients in the ward.

(9) Have you encountered any disturbing experiences when collecting patients from the theatre?

FIG 1-Questions the nurses were asked.

The answers to the first six questions were compared within the two groups: between SRNs and SENs in group 1 and between student and pupil nurses in group 2 . The answers were labelled correct or incorrect, the latter including "unknown" answers. The number of incorrect answers was expressed as a percentage of the total answers for each question. Data were analysed using the $\chi^{2}$ test with Yates's correction or Fisher's exact test when small numbers were used.

Another 116 nurses were asked to give the approximate point of their training when they were first asked to collect a patient from the operating theatre. They were asked to state whether or not they thought that they were sufficiently experienced for this task. Again, the questionnaire was conducted on a random, voluntary, and confidential basis. This group included nurses who had trained in other regions, and the results might therefore be more representative of practice generally.
RESULTS

The results for questions 1-6 in the groups of trained nurses and trainees are shown in table I.

Group 1-SENs provided the greater proportion of incorrect answers except for question $6 a$. There was no significant difference between the proportion of incorrect answers provided by SRNs and SENs to questions $1,2,3$, or 6 , but there was a significant difference $(P=0.05)$ between the proportions of incorrect answers given to questions 4 and 5 , with the SENs giving significantly more incorrect answers.

Group 2-The pupil nurses provided the greater proportion of incorrect answers to all questions. There was no significant difference between the proportions of incorrect answers to questions $1,2,3$, and $6 b$, but there was a significant difference between the proportion of incorrect answers given to questions $4(P=0.01), 5(P=0.01)$, and $6 a$, with the pupils showing a significant number of incorrect answers in each case.

The results to questions 7-9 are shown in tables II and III.

TABLE II-Results of questions 7 and 8 in trained nurses and trainees

\begin{tabular}{|c|c|c|c|c|}
\hline & \multicolumn{2}{|c|}{ Group 1} & \multicolumn{2}{|c|}{ Group 2} \\
\hline & SRNs & SENs & Students & Pupils \\
\hline $\begin{array}{l}\text { Question } 7 \\
\text { (a) Conscious } \\
\text { (b) Semiconscious } \quad . \\
\text { (c) Unconscious } \quad \ldots \\
\text { Question } 8 \\
\text { Satisfactory answer.. } \\
\text { Unsatisfactory answer }\end{array}$ & $\begin{array}{r}36 \\
9 \\
0 \\
54 \\
1\end{array}$ & $\begin{array}{r}18 \\
18 \\
0 \\
33 \\
3\end{array}$ & $\begin{array}{r}24 \\
17 \\
1 \\
42 \\
0\end{array}$ & $\begin{array}{r}13 \\
17 \\
0 \\
28 \\
2\end{array}$ \\
\hline
\end{tabular}

TABLE III-Answers to question 9

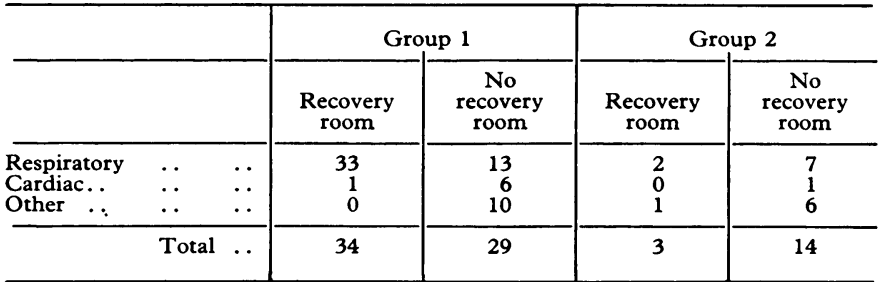

One hundred and seven nurses gave information about the point during their training when they were first asked to collect a patient from the operating theatre. They were also asked whether or not they felt confident for this task. The results are as shown in table IV.

TABLE IV-Nurses' answers about the time when they first collected postoperative patients and how confident they felt

\begin{tabular}{r|c|c|c|c}
\hline Time in training (months): & $<3$ & -6 & -12 & $>12$ \\
\hline $\begin{aligned} \text { No }(\%) \text { who felt confident } \ldots \\
\text { No }(\%) \text { who did not feel confident }\end{aligned}$ & $\begin{array}{r}0 \\
22(100)\end{array}$ & $\begin{array}{r}4(17) \\
19(83)\end{array}$ & $\begin{array}{r}15(44) \\
19(56)\end{array}$ & $\begin{array}{r}20(71) \\
8(29)\end{array}$ \\
\hline Total.. & 22 & 23 & 34 & 28 \\
\hline
\end{tabular}

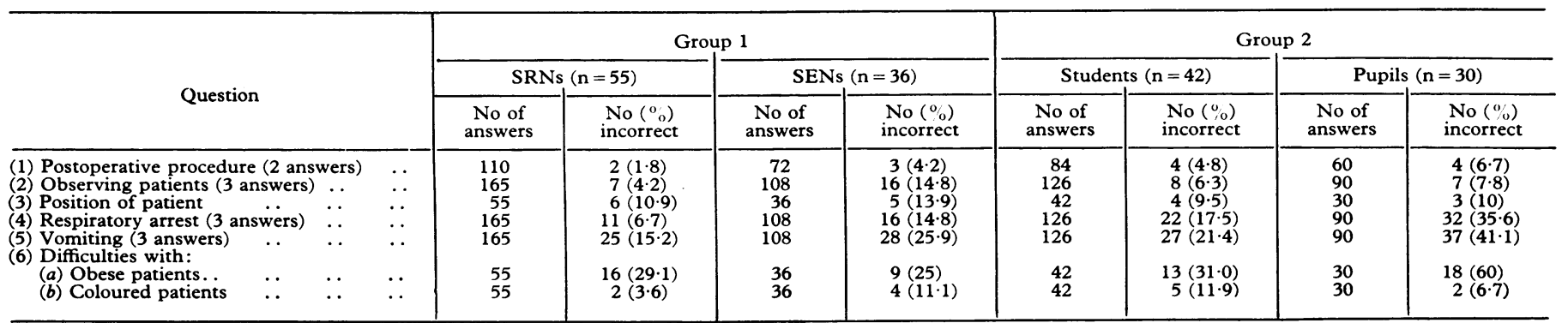




\section{Recovery facilities}

Consultant anaesthetists in the South-east Thames Region were asked to provide information on the recovery facilities in the main surgical units where they worked and to say whether they were satisfied with these facilities. The questions asked are shown in fig 2 .

FIG 2-Questions the consultant anaesthetists were asked.

(1) Number of operating theatres.

(2) Number of recovery rooms/areas.

(3) Do you have a special room?

(4) Do you use a corridor?

(5) Others-please specify.

(6) Lighting of recovery area:

(a) Good daylight or fluorescent "daylight" quality.

(b) Adequate.

(c) Poor.

(7) Suction:

(a) Immediately to hand.

(b) In adjacent room.

(8) Oxygen:

(a) Immediately to hand.

(b) In adjacent room.

(9) Communication:

(a) Bell-position in relation to nurse. Is it checked regularly ?

(b) Calling for help.

(c) Other.

(10) Tipping trolley:
(a) All cases.
(b) Some cases.
(c) Rare.

(11) Staff allocation:

(a) 1 nurse : 1 patient.
(b) 1 nurse : 2 patients.

(b) 1 nurse

(12) Are there differences in procedure after normal working hours in:

(a) Facilities-for example, closure of recovery rooms.

(b) Staff allocation.

(13) Do you have special facilities for day cases?

(14) Are you satisfied with immediate postoperative recovery facilities in your surgical units, and if not, can you suggest how they may be improved?

\section{RESULTS}

Twenty-two replies were received giving information on recovery facilities in 27 surgical units in the South-east Thames Region. The answers to questions 1-11 are shown in table V.

Question 12: differences after normal working hours-Facilities at night were generally thought to be adequate despite the fact that

TABLE V-Consultants' answers to questionnaire

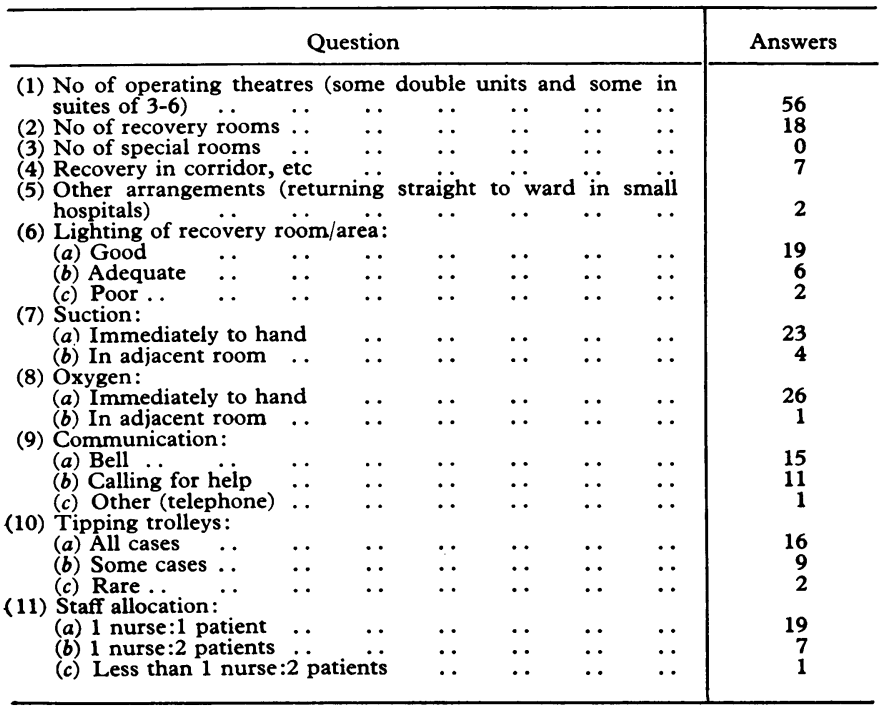

recovery rooms were usually closed. Staff shortages proved to be the main continuing problem. In spite of this patients were usually kept in theatre until they regained consciousness before being returned to possibly even more inadequately staffed wards.

Question 13: day-case facilities-Six hospitals had day-case or short-stay wards, but in only three did they include provision for postoperative recovery.

Question 14: degree of satisfaction-Thirteen consultants said that they were generally satisfied with the facilities where they worked, and 14 said that they were not. Three of these who were dissatisfied were waiting for the new hospitals to be opened within the next two years. One consultant anaesthetist said that the only solution would be a new hospital, as yet only a pipe dream. All those expressing dissatisfaction put staffing at the head of the list of priorities for upgrading.

\section{Discussion}

The nurses' replies to the first three questions on their questionnaire were satisfactory, which indicates that nurses are competent to care for the unconscious patient so long as an emergency does not arise. Question 8, which dealt with the care of the unconscious patient on the ward, was also well answered. This suggests that nurses feel more confident in the stable atmosphere of the ward, where they would be more likely to be caring for patients in prolonged coma than after an operation, when the patient is emerging from general anaesthesia. The high proportion of incorrect or "missed" answers to questions 4 and 5, which dealt with the common postanaesthetic emergencies, tendes to confirm this view. In both groups 1 and 2 there was a significant difference between the SRNs and SENs in the replies to questions 4 and 5, and in group 2 this also applied to question $6 a$. This was expected as a higher standard of training is demanded for State registration, but SRNs and SENs have a similar part to play in this essentially practical aspect of nursing, so this difference should be minimised by more emphasis being placed on the subject in the SEN's syllabus. Question $6 a$ referred to the ever-increasing problem of obesity, which adds to the morbidity and mortality rate after operation. Nurses are obviously not sufficiently aware of the difficulty of maintaining a clear airway in obese people or of how obesity may mask the early recognition of airway obstruction, particularly in the supine position. Nurses more readily appreciated the difficulty of recognising cyanosis in coloured patients, though many had to think about the question, and it was clear that this problem had rarely been brought to their attention before.

Most training about the care of an unconscious patient is given in the introductory course, which means that at least six months intervenes before trainees are expected to put this teaching into practice in caring for postanaesthetic patients. This corresponds with the accounts of their training given by nurses who answered the questionnaire. They remembered the early teaching in the introductory course but subsequent practical training tended to be variable and erratic.

Training on the ward obviously depends on the availability of trained staff, the aptitude of the ward sister, and the amount of time she can devote to teaching. The present shortage of nurses means that the service commitment expected of trainees is far too high, and they are sometimes asked to collect patients from the operating theatre before they feel confident to do so. Of the 107 nurses who replied to our second questionnaire and gave information on the point in their training at which they were first asked to collect a patient, well over $40 \%$ admitted to having done this within the first six months of their training and $20 \%$ within the first three months. Officially a learner should have 12 months' nursing experience before collecting a postoperative patient unaccompanied, particularly in hospitals with no recovery room. Our inquiry confirmed that a nurse feels fully confident only after 12 months of training.

The replies to question 7 showed that the more experienced the nurse, the more likely he or she is to prefer to return a 
postoperative patient to the ward when fully conscious. Over $60 \%$ of the SRNs said that they preferred this. The other groups were divided more equally in their preference to return patients either semiconscious or fully conscious. Only one nurse was happy to return an unconscious patient.

\section{RECOVERY FACILITIES}

The answers to question 9 confirm the value of recovery rooms. By far the most emergencies occurred in patients who were still unconscious in hospitals with no recovery rooms. Nurses have vivid memories of these incidents, which are obviously very frightening, particularly for learners. The feeling of not clearly knowing what to do in an emergency accounted for many nurses' lack of confidence. Most of the emergencies were episodes of respiratory arrest or depression, and haemorrhage was not mentioned as a postoperative problem in this survey-a compliment to modern surgery.

The fact that nurses are being asked to take on the responsibility for the postoperative care of patients alone at an increasingly early stage in their training is compounded in many cases by the inadequacy of the facilities provided. Although the financial stringencies of the NHS have delayed and will continue to delay the provision of fully equipped and staffed recovery rooms, there seems to be little excuse for some inadequacies. For example, in 12 of the 27 surgical units considered in our third questionnaire help could be obtained in an emergency only by shouting for help or knocking on the theatre door. The fact that suction was not immediately to hand in four units and oxygen not immediately available on the patient's trolley in one case is equally unsatisfactory.

\section{Conclusions}

A fully equipped recovery bay or area close to expert help is mandatory in all busy surgical units. The cost of such a room should not be excessive, requiring only good communications, adequate lighting, and a source of oxygen and suction. Only in a "cottage" hospital, where minor surgery is carried out, it is reasonable to return the patients straight to the ward, which is usually close to the theatre. It may then be desirable to nurse the patient in the lateral position as soon as he is returned to bed.

\section{POSTOPERATIVE NURSE}

The shortage of nursing staff is likely to remain chronic while NHS resources are limited. It is therefore vital to make the most effective use of currently available trained nursing staff. Using a ward nurse, no matter how well-trained, is a poor compromise. Her departure to collect a patient after operation will deplete the ward staff (usually very busy on an operation day), so she will be reluctant to stay near the theatre until the patient has fully recovered from anaesthesia. She may be tempted to return the patient before it is safe for him to make the journey, or she may try to wake the patient forcibly, which is most undesirable. All busy surgical units should therefore have a trained nurse whose sole responsibility is to care for postoperative patients until they are fully conscious. One nurse should be provided for every one patient or, at most, two patients.

\section{TRAINING}

It should be recognised that trainee nurses might be expected to collect postoperative patients fairly early in their training, perhaps after the first six months. Further demonstrations or a refresher course on the care of the unconscious postanaesthetic patient should be given after five to six months of training rather than concentrating all such training into the introductory course. Practical refresher courses should be provided from time to time for trained nurses, particularly those who have been away from nursing for some time. More emphasis should be given to using resuscitation equipment such as oxygen and suction. A surprising number of nurses forget to open the Acidbrink valve between the mask and the reservoir bag when administering oxygen. Many nurses said that they expected to use the gauze and sponge holder to remove vomit rather than suction.

Although nurses should be trained to use the emergency instruments they take with them to the ward (mouth gag, tongue depressor, and sponge holder) we hope that they may never be required to use them. All nurses should be competent at administering oxygen and removing secretions and vomit by suction. More emphasis should also be given to the particular problems likely to be encountered in certain types of patientsfor example, the obese, the coloured patient, and very young or very old patients.

Nurses in charge of recovery should be able to help the clinical tutors in teaching, and anaesthetists should also be prepared to help in the practical aspects of training. It is vital that anaesthetists should show an active interest in the stage of emergence from anaesthesia, so that nurses can feel confident that help will always be there when needed. Continuous co-operation between anaesthetists and nursing tutorial staff is essential to ensure that the training programme fulfils and continues to fulfil the requirements of the particular district.

We would like to acknowledge the help of the following in the preparation of this paper: the nursing staff who helped to draft the nurse questionnaire, the nurses who took part in the questionnaire, and the consultant anaesthetists who provided information on recovery facilities. We should also like to thank Mrs S Smith, Mrs R Clifford, and Mrs M Fisher for their secretarial help.

\section{References}

1 Registrar General's Statistical Review, pt I Tables, Medical, 1950-1970, London, HMSO, 1952-72.

${ }^{2}$ Wylie, W D, Annals of the Royal College of Surgeons, 1975, 56, 175.

${ }^{3}$ Department of Health and Social Security, Hospital Building Recommendation, C9 SFB 4025 26, London, DHSS, 1967.

${ }^{4}$ Operating theatre records, St Bartholomew's Hospital, Rochester, 1972.

(Accepted 12 February 1977)

\section{What is the present treatment of herpes genitalis?}

Unfortunately there is no curative treatment. Local applications of idoxuridine and other preparations have proved disappointing both in shortening the duration of individual attacks and in preventing recurrences. This is not surprising, as it is now well established that the virus invades the nerve sheaths and the posterior root ganglia during the primary attack, and to eradicate it a systemic antiviral agent is required. ${ }^{1}$ At present there is no such preparation of proved value. Treatment consists of keeping the lesions clean, and the regular application of physiological saline solution is probably as satisfactory as anything else and is harmless. If there is severe pain appropriate analgesics should be given, and when the lesions become secondarily infected a sulphonamide or co-trimoxazole by mouth at the usual dosage for five or seven days is often helpful. Treponemocidal antibiotics are better avoided. The recurrent nature of the condition should be explained to the patient, who should also be told of the risk of infecting a sexual partner when active open lesions are present. Women with genital herpes should be advised to have regular annual cervical cytology. In many patients the attacks become progressively less frequent and less severe and often cease after a few years. Moderate optimism about the long-term prognosis is therefore reasonable as many patients become depressed by frequent recurrences of the lesions.

${ }^{1}$ Baringer, J R, New England fournal of Medicine, 1974, 291, 823. 